\title{
Incidence of hypomagnesemia on proton pump inhibitors at the Huntington Veterans Affairs Medical Center - IHOP
}

Ebrahim Sabbagh ${ }^{1}$, Chelsey R Houchins ${ }^{2}$, James Allman II ${ }^{2}$, Samson Teka ${ }^{1}$

\section{Author Affiliations:}

1. Marshall University

2. Veterans Affairs Medical Center

The authors declare no actual or potential conflicts of interest.

\section{Corresponding Author:}

Ebrahim Sabbagh, D.O.

Internal Medicine Chief Resident

Marshall University Joan C. Edwards School of Medicine

Huntington, West Virginia

Email: sabbaghe@marshall.edu 


\section{Abstract}

\section{Purpose}

Proton pump inhibitors (PPIs), both prescription and over-the-counter, are widely used for the treatment of acid-related disease states such as dyspepsia, gastroesophageal reflex disease, esophagitis, and peptic ulcers. These medications are generally considered safe in most patient populations; however, there are several adverse effects that may occur with long-term use. Hypomagnesemia is a newer complication, arising in the literature following multiple case reports over the past several years; the true incidence of hypomagnesemia associated with PPI use, however, remains unclear at this time.

\section{Methods}

A retrospective chart review was performed on patients enrolled at the Huntington Veterans Affairs Medical Center (VAMC) who are receiving or have received long-term PPI therapy (considered $\geq 3$ months). Magnesium levels were reviewed to determine the incidence of hypomagnesemia with PPI use. Other data analyzed included age, PPI prescribed, calcium level, potassium level, and diuretic use (both Loop and thiazide).

\section{Results}

Three hundred thirty one patients were identified and reviewed that had a prescription for a PPI for 3 months or longer, between 2000 and 2014. Of the 331 patient charts reviewed, 192 met the study inclusion criteria and were analyzed for hypomagnesemia while on PPI therapy. There was a total of 51 out of 192 patients $(26.6 \%)$ with hypomagnesemia reported at least 3 months after starting PPI therapy. The decline in magnesium levels over time while on PPI therapy resulted in a Pearson's Correlation of -0.24 which was statistically significant $(\mathrm{p}<0.001)$. The average age of patients was similar between those with a low magnesium compared to those with a normal magnesium, 65.9 years old and 67.4 years old, respectively $(\mathrm{p}=0.33)$. Serum potassium and calcium levels were almost identical in those with hypomagnesemia compared to those with a normal magnesium level ( $\mathrm{p}=0.38$ and $\mathrm{p}=0.57$, respectively). Diuretic use was higher in those with a low magnesium at $62.7 \%$ compared to $53.9 \%$ in those with a normal magnesium, although this was not statistically significant $(\mathrm{p}=0.28)$. When magnesium levels in those without diuretic therapy were reviewed, a Pearson's correlation of -0.18 was found $(p=0.10)$. A stronger correlation was found if a patient was on diuretic therapy which resulted in a Pearson's Correlation of -0.32 and this was statistically significant $(\mathrm{p}=0.001)$. Using a logistic regression model, it was estimated that each month of combination PPI and diuretic therapy resulted in a $1.5 \%$ higher risk of hypomagnesemia.

\section{Conclusion}

In conclusion, hypomagnesemia may result in patients on long-term PPI therapy regardless of age and diuretics may further contribute to this issue. Low magnesium does not appear to be correlated with hypokalemia or hypocalcemia in this patient population. Baseline and routine monitoring of magnesium should be considered in patients that are being started on PPI therapy or are continued on therapy long-term. Additional studies would be helpful in determining the incidence of hypomagnesemia with the addition of a PPI in patients already prone to this 
problem. The use and effectiveness of magnesium supplementation in those with hypomagnesemia while on a PPI should be evaluated to determine if patients should be on longterm supplementation or if alternative antacid therapy should be considered.

\section{Keywords}

Hypomagnesemia, proton pump inhibitors

\section{Introduction and Background Information}

Proton pump inhibitors (PPIs), both prescription and over-the-counter, are widely used for the treatment of acid-related disease states such as dyspepsia, gastroesophageal reflex disease, esophagitis, and peptic ulcers. These medications are generally considered safe in most patient populations. Potential mild, self-limiting side effects include abdominal pain, flatulence, constipation, and diarrhea; however, there are several adverse effects that may occur with longterm use. These include nutritional deficiencies such as calcium, iron, and vitamin B12, pneumonia or Clostridium difficile infections, and even an increased risk of colon and gastric cancers. Hypomagnesemia is a newer complication arising in the literature following multiple case reports over the past several years; the true incidence of hypomagnesemia associated with PPI use, however, is still unclear at this time.

Magnesium is the second most abundant intracellular cation in the body. Magnesium homeostasis is maintained through gastrointestinal absorption and renal excretion.

Gastrointestinal absorption involves both passive and active movement of magnesium. A serum magnesium level is often used to determine a person's magnesium level within the body and determine if supplementation is needed; however, roughly $0.3 \%$ of total magnesium is found within the serum. The overwhelmingly majority of magnesium is found in bone, muscle, and non-muscular soft tissue and accounts for about $99 \%$ of total body stores. Serum magnesium can be helpful for detecting rapid extracellular changes of magnesium. ${ }^{1}$

Hypomagnesemia associated with PPI use was first described in 2006 involving two case reports. ${ }^{2}$ After receiving continuous case reports over the past years, the FDA issued a warning in 2011 announcing the association between long-term PPI use and hypomagnesemia. ${ }^{3}$ PPIs are thought to affect passive absorption by disrupting the transient receptor potential melastatin- 6 and -7 channels (TRPM6/7). This disruption is believed to be caused by affecting the transport channels directly and/or altering gastrointestinal $\mathrm{pH}$ causing a reduction of the ionized vs. unionized ratio, ultimately decreasing the affinity and absorption of magnesium by TRPM6/7 channels . ${ }^{4,5}$ Hypomagnesemia from use of PPIs is considered a long-term complication that can take months or years to develop with no clear dose relationship. Most case reports occurred after one year of PPI treatment, but there are some reports of hypomagnesemia occurring in patients as early as 3 months after starting therapy. ${ }^{5}$

Recent studies involving PPIs and magnesium levels have led to slightly conflicting results. Danziger et al reviewed 2,632 adult ICU admissions in patients on PPI therapy. They determined that low magnesium was limited to those who were also on diuretic therapy. ${ }^{6}$ Koulouridis et al reviewed 402 adult admissions who had hypomagnesemia upon arrival. They concluded out-ofhospital PPI use was not associated with low magnesium level. ${ }^{7}$ Luk et al reviewed 66,102 adult 
admissions and found 693 patients on PPI therapy who also had a low magnesium. They estimated PPI-associated hypomagnesemia to be roughly $1 \%$ and that this risk was increased in males and the elderly population. They also concluded that it was associated with hypocalcemia and hypokalemia. ${ }^{8}$ Most recently, Zipursky et al compared 366 patients hospitalized with low magnesium to 1,464 matched controls. They determined that concomitant PPI and diuretic therapy lead to a small increased risk of hospitalization with hypomagnesemia. ${ }^{9}$

Low magnesium can cause both minor and serious complications depending on the severity. Symptoms may range from gastrointestinal upset, weakness, and cramping to metabolic abnormalities, cognitive changes, seizures, and arrhythmias. Although most mild cases go unnoticed, more severe cases could result in life-threatening events. Magnesium is not a component of the electrolyte 7 panel most commonly drawn at the Huntington VAMC. Magnesium requires a separate order and is often checked when a patient's level is expected to be out of the normal range. The association between PPIs and low magnesium may warrant more frequent monitoring in patients on long-term therapy.

\section{Methods}

A retrospective chart review was performed on patients entrolled at the Huntington VAMC who are receiving or have received long-term PPI therapy (considered $\geq 3$ months). Magnesium levels were reviewed to determine the incidence of hypomagnesemia with PPI use. Hypomagnesemia was determined to be less than $1.8 \mathrm{mg} / \mathrm{dl}$ (normal range 1.8 to $2.4 \mathrm{mg} / \mathrm{dl}$ ). Other data analyzed included age, PPI prescribed, calcium level, potassium level, and diuretic use (both Loop and thiazide). Hypokalemia was determined to be less than $3.5 \mathrm{mmol} / \mathrm{L}$ (normal range 3.5-5.0 $\mathrm{mmol} / \mathrm{L}$ ) and hypocalcemia was determined to be less than $8.4 \mathrm{mg} / \mathrm{dl}$ (normal range 8.4-10.7 $\mathrm{mg} / \mathrm{dl})$.

The primary endpoint of the study was to determine the incidence of hypomagnesemia with long-term PPI therapy. Secondary endpoints included the association of hypoglycemia based on patients' age, association with hypokalemia and/or hypocalcemia, and association with diuretic use. Patients were included in the study if they were 18-89 years of age, had a PPI prescription for at least 3 months, and had a magnesium level reported at least 3 months after starting PPI therapy. Patients were excluded from the study if they were on magnesium supplementation or had one of the following: abdominal surgery, acute or chronic diarrhea, alcoholism, chronic inflammatory bowel disease, end-stage renal disease requiring dialysis or transplant, pancreatitis, or primary hypoparathyroidism as these alone may place a patient at risk of hypomagnesemia.

\section{Results}

Three hundred thirty one patients were identified and reviewed that had a prescription for a PPI for 3 months or longer. Of the 331 charts reviewed, 139 patients were excluded based on the reasons listed in Figure 1. 
Figure 1. Veterans Affair chart reviewed.

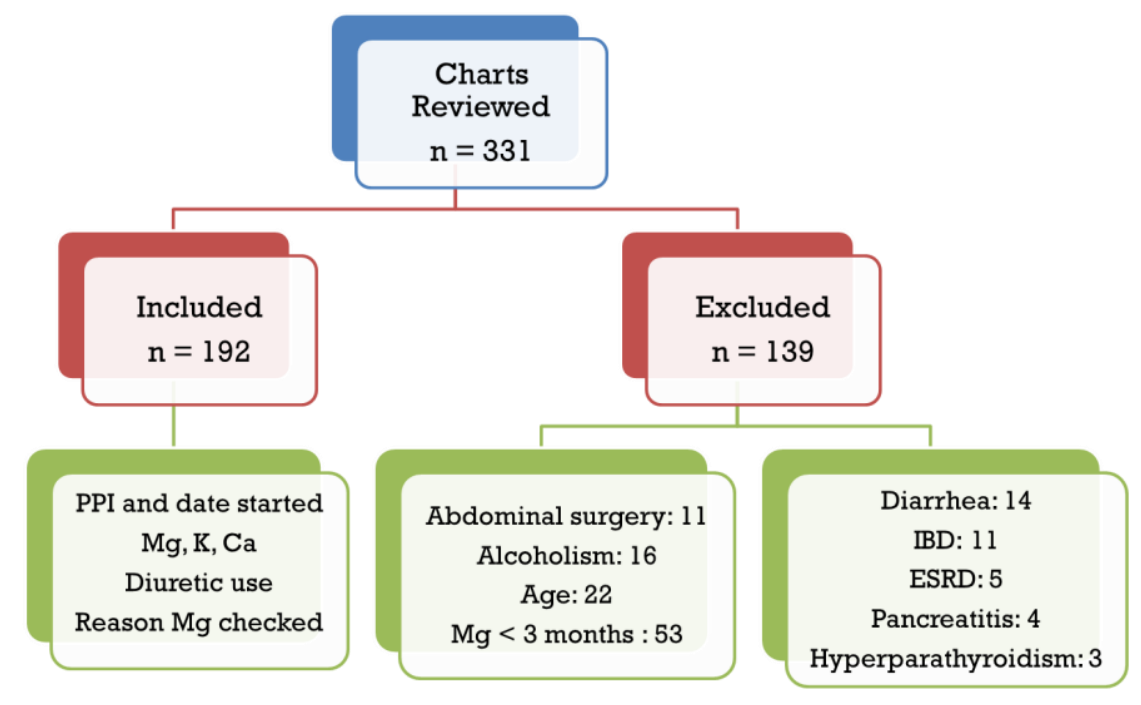

The remaining 192 patients met the study inclusion criteria and were analyzed for hypomagnesemia while on PPI therapy. The most common PPI utilized was omeprazole at $85.9 \%$ (Table 1). A total of 121 concomitant diuretics were used and are shown in Table 2. Fifty one percent of patients were on one Loop or thiazide diuretic at the time serum magnesium was obtained and only $5.2 \%$ of patients were on two diuretics (Table 3 ).

Table 1. Number of Patients on Different Types of PPI

\begin{tabular}{|c|c|}
\hline Type of PPI $(\mathrm{n}=192)$ & Number $(\%)$ \\
\hline Omeprazole & $165(85.9)$ \\
\hline Pantoprazole & $14(7.3)$ \\
\hline Lansoprazole & $8(4.2)$ \\
\hline Rabeprazole & $4(2.7)$ \\
\hline Esomeprazole & $1(0.5)$ \\
\hline
\end{tabular}

Table 2. Number of Patients on Diuretics and Type.

\begin{tabular}{|c|c|}
\hline Type of Diuretics $(\mathrm{n}=121)$ & Number $(\%)$ \\
\hline Furosemide & $61(50.4)$ \\
\hline Hydrochlorothiazide & $59(48.8)$ \\
\hline Bumetanide & $1(0.8)$ \\
\hline
\end{tabular}


Table 3. Number of Patients on Diuretic.

\begin{tabular}{|c|c|}
\hline Number of Diuretics $(\mathrm{n}=192)$ & Number $(\%)$ \\
\hline None & $84(43.8)$ \\
\hline One & $98(51.0)$ \\
\hline Two & $10(5.2)$ \\
\hline
\end{tabular}

The average time to collection of magnesium was 46 months after starting a PPI. Common reasons a patient's serum magnesium was checked include cardiac abnormalities, hypokalemia, cramps, generalized weakness, seizure activity, history of alcoholism, or routine clinic visits. There were a total of 51 out of 192 patients (26.6\%) with hypomagnesemia reported at least 3 months after starting PPI therapy (Figure 2).

Figure 2. Incidence of Hypomagnesemia

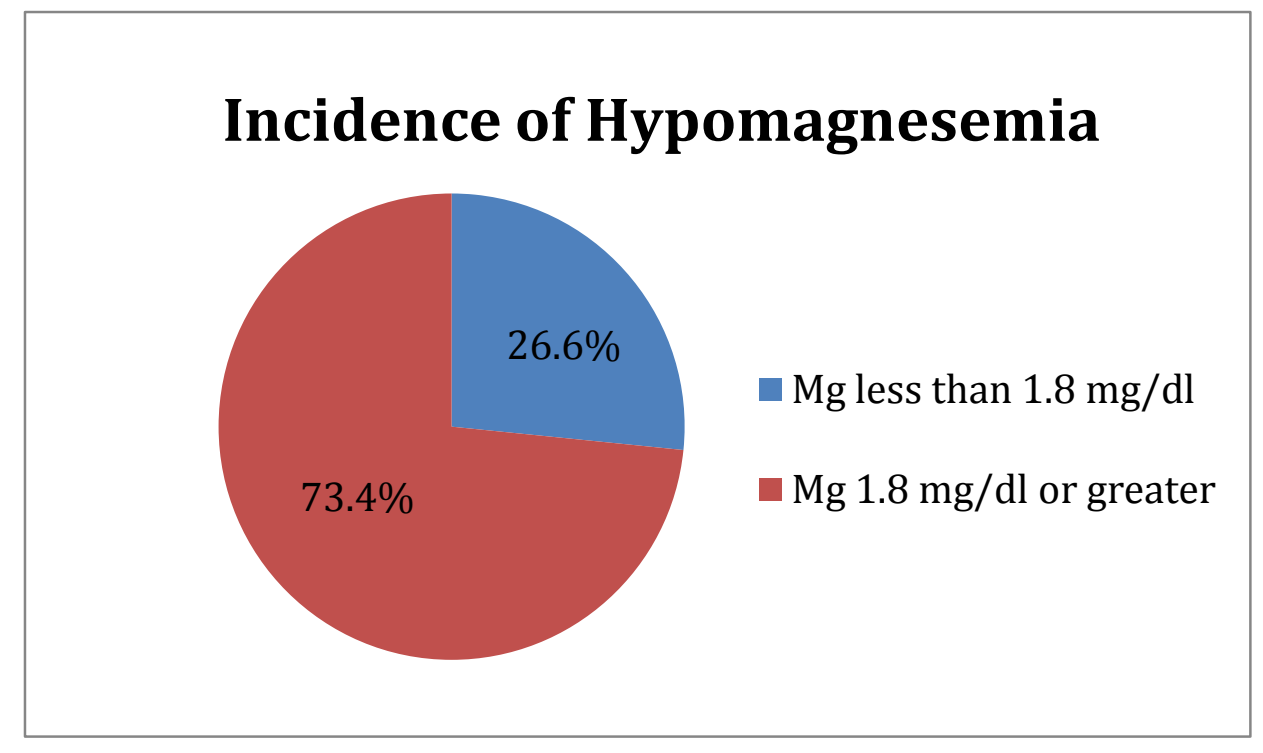

Figure 3 illustrates the decline in magnesium levels over time while on PPI therapy which resulted in a Pearson's Correlation of -0.24 which was statistically significant $(\mathrm{p}<0.001)$. Secondary endpoints comparing patients with low magnesium to those with normal magnesium can be found in Table 4 . The average age of patients was similar between those with a low magnesium compared to those with a normal magnesium, 65.9 years old and 67.4 years old, respectively $(\mathrm{p}=0.33)$. Serum potassium and calcium levels were almost identical between the two groups and was also not statistically significant ( $\mathrm{p}=0.38$ and $\mathrm{p}=0.57$, respectively). Diuretic use was higher in those with a low magnesium at $62.7 \%$ compared to $53.9 \%$ in those with a normal magnesium, although this was not statistically significant $(\mathrm{p}=0.28)$. 
Figure 3. Hypomagnesemia on PPI Therapy Over Time

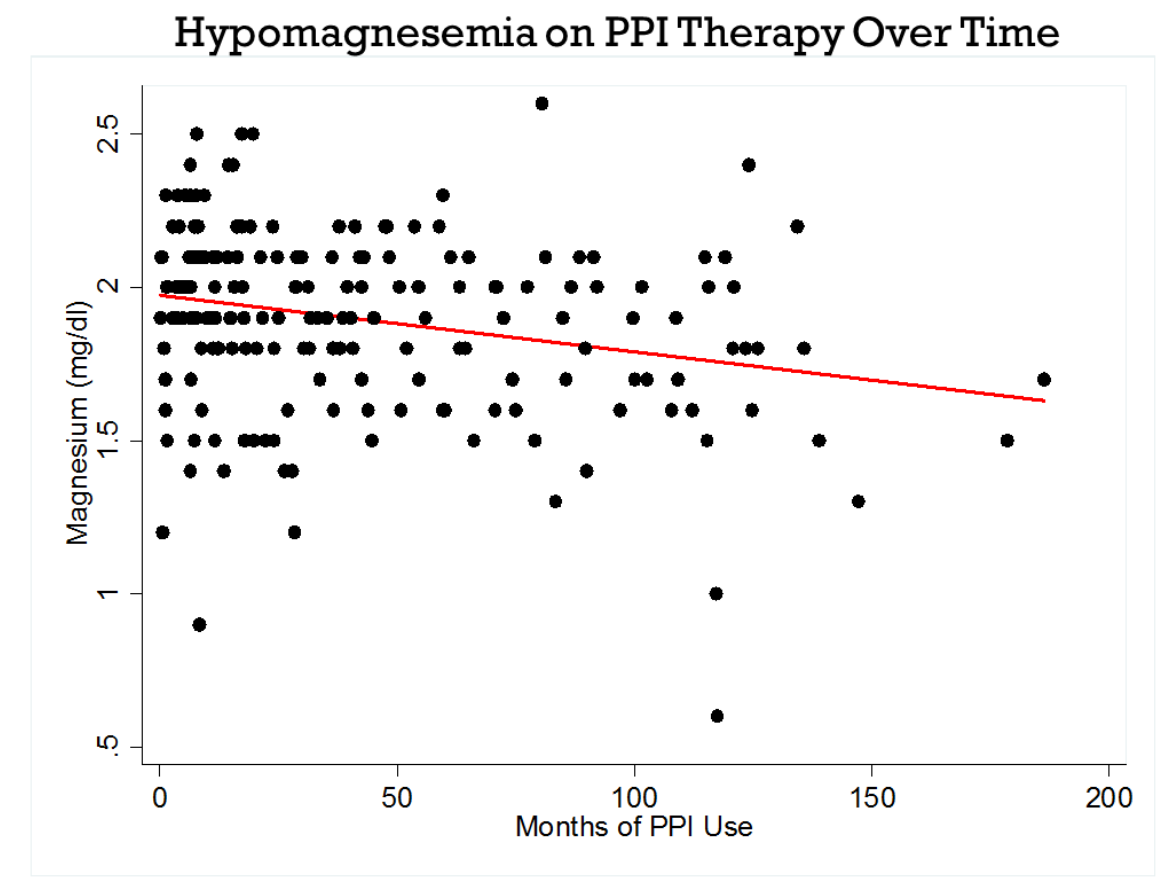

Pearson's Correlation $=-0.24(\mathrm{p}<0.001)$

Table 4. Secondary Endpoints Comparing Patients with Low Magnesium to Those with Normal Magnesium

\begin{tabular}{|c|c|c|c|}
\hline Characteristic & $\begin{array}{c}\text { Low Magnesium } \\
(\mathrm{n}=51)\end{array}$ & $\begin{array}{c}\text { Normal Magnesium } \\
(\mathrm{n}=141)\end{array}$ & p Value \\
\hline Age, mean (sd) & $65.9(9.3)$ & $67.4(10.0)$ & 0.33 \\
\hline Potassium, mean (sd) & $4.3(0.54)$ & $4.2(0.58)$ & 0.38 \\
\hline Calcium, mean (sd) & $9.1(0.68)$ & $9.2(0.62)$ & 0.57 \\
\hline Diuretics, N (\%) & $32(62.7)$ & $76(53.9)$ & 0.28 \\
\hline
\end{tabular}

Magnesium levels and diuretic use was further analyzed and is illustrated in Figure 4. When magnesium levels in those without diuretic therapy were reviewed, a Pearson's correlation of 0.18 was found $(\mathrm{p}=0.10)$. A stronger correlation was found if a patient was on diuretic therapy which resulted in a Pearson's Correlation of -0.32 and this was statistically significant $(\mathrm{p}=0.001)$. 
Using a logistic regression model, it was estimated that each month of combination PPI and diuretic therapy resulted in a $1.5 \%$ higher risk of hypomagnesemia.

Figure 4. Comparing Magnesium Levels with Diuretic Use

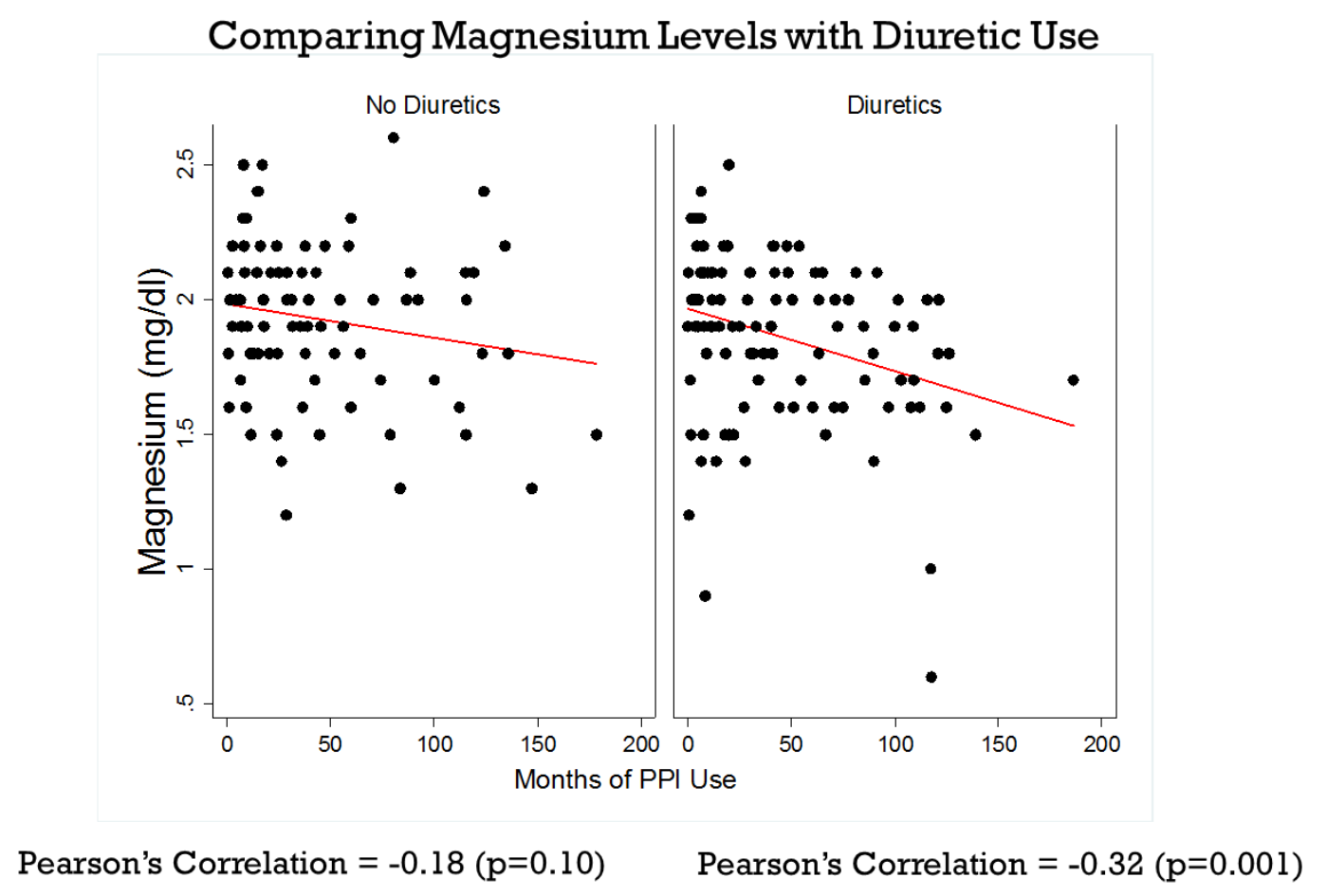

\section{Discussion}

When reviewing patients on PPI therapy for 3 months or longer, there was a weak correlation with hypomagnesemia regardless of age. This effect appears to happen over time regardless of diuretic use, but this was stronger when a concomitant diuretic therapy is used.

Hypomagnesemia did not appear to be correlated with hypokalemia or hypocalemia in these specific patients.

An intrinsic factor to keep in mind is that serum magnesium levels were often checked by providers when the patient was having potential symptoms related to low levels. The majority of patients were excluded due to no magnesium level after initiation of PPI therapy.

There are several limitations to this study in extrapolating results to the general population since the majority of the patients within the VA health system are middle-aged to elderly males. Also, those patients already prone to low magnesium levels were excluded in the study so the addition of PPI therapy to these types of patients was not analyzed. Patient compliance could not be determined due to the retrospective nature of the study. There were three data collections for the study which could contribute to some variability; however, this was minimized with a standardized data collection sheet. Baseline serum magnesium levels were not utilized and would have been useful to determine if certain patients had low magnesium levels prior to PPI use. The 
study population was smaller due to limited magnesium levels reported since it is not checked in most patients as part of most routine laboratory collections (i.e. electrolyte 7 panel). A larger study population may yield more robust results and may detect more significant correlations.

\section{Conclusion}

In conclusion, hypomagnesemia may result in patients on long-term PPI therapy regardless of age. Diuretics may further contribute to this issue. Low magnesium does not appear to be correlated with hypokalemia or hypocalcemia in this patient population. Baseline and routine yearly monitoring of magnesium should be considered in patients that are being started on PPI therapy or are continued on therapy long-term. Additional studies would be helpful in determining the incidence of hypomagnesemia with the addition of PPI therapy in patients already prone to this problem or continued on therapy long-term. The use and effectiveness of magnesium supplementation in those with hypomagnesemia while on a PPI should be evaluated to determine if patients should be on long-term supplementation. Patients should also be carefully evaluated to see long term PPI is really indicated, or if alternative antacid therapy can be considered. Patients whom have uncontrolled GERD without long term PPI should be evaluated for surgical or endoscopic treatment.

\section{Acknowledgements}

Dr. Todd Gress, M.D., MPH for help with statistical analysis of the research data.

Dr. Dennis Lester, M.D. for help in gathering data. 


\section{References}

1. Reimer C, Sonderaard, Hilsted L, Bytzer P. Proton-pump inhibitor therapy induces acid-related symptoms in healthy volunteers after withdrawal therapy. Gastroenterology 2009;137(1):80-87.

2. Epstein M, McGrath S, Law F. Proton-pump inhibitors and hypomagnesemic hypoparathyroidism. N Engl J Med 2006;355:1834-1836.

3. U.S. Food and Drug Administration [www.fda.gov]. Center for Drug Evaluation, Research. FDA Drug Safety Communication: low magnesium levels can be associated with long-term use of proton pump inhibitor drugs (PPIs) [updated 14 March 2011]. Centers for Drug Evaluation and Research. Available from URL: http://www.fda.gov/drugs/drugsafety/ucm245011.htm.

4. Perazella MA. Proton pump inhibitors and hypomagnesia: a rare but serious compliacation. Kidney International 2013;83:553-556.

5. Florentin M, Elisaf MS. Proton pump inhibitor-induced hypomagesemia: a new challenge. World Journal of Nephrology 2011;1(6):151-154.

6. Danziger J, et al. Proton-pump inhibitor use is associated with low serum magnesium concentrations. Kidney International 2013;83:692-699.

7. Koulouridis I, et al. Out-of-hospital use of proton pump inhibitors and hypomagesemia at hospital admission: A nested case-control study. Am J Kidney Dis 2013;62(4):730-737.

8. Luk CP, Parsons R, Lee YP, Hughes JD. Proton pump inhibitor-associated hypomagesemia: what do FDA data tell us? The Annals of Pharmacotherapy 2013;47:773-780.

9. Zipursky J, et al. Proton pump inhibitors and hospitalization with hypomagnesemia: a population-based casecontrol study. PLOS Medicine 2014;11(9):1-7. 